\title{
Completion Node Dissection for Sentinel Node-Positive Melanoma: Can a Systematic Review Bring One Discussion to a Close While Leaving the Broader Conversation Still Open?
}

\author{
Russell S. Berman, $\mathrm{MD}^{1}$ and Jeffrey E. Gershenwald, $\mathrm{MD}^{2}$ \\ ${ }^{1}$ Department of Surgery, Division of Oncology, New York University School of Medicine, New York, NY; ${ }^{2}$ Division of \\ Surgery, Department of Surgical Oncology, The University of Texas MD Anderson Cancer Center, Houston, TX
}

The Annals of Surgical Oncology usually does not commission an editorial focused on an educational review article. However, in this editorial, we have been asked to do just that. In this editorial, we explore beyond the clinical content of "The Role of Completion Lymph Node Dissection (CLND) for Sentinel Lymph Node (SLN)-Positive Melanoma" by Hieken and colleagues, and consider the process and methods used to perform this review. ${ }^{1}$

The initial results of two randomized phase 3 clinical trials designed to explore the role of CLND in patients with a tumor-involved SLN-the DeCOG-SLT ${ }^{2}$ and the Multicenter Selective Lymphadenectomy II (MSLT-II) ${ }^{3}$ - have inspired numerous commentaries, from the editorial published concomitantly with MSLT-II ${ }^{4}$ to the recent editorial by Rand and Faries ${ }^{5}$ in this journal. Importantly, our objective is not to debate further the role of CLND in SLNpositive melanoma, nor is it to delineate clinical guidelines. Rather, we aim to illuminate the potential advantages and limitations of a well-performed systematic review.

Although it is appropriate to prioritize relevant and wellexecuted prospective, randomized clinical trials as key components of the evidence base, a well-conducted systematic review also may play a role in helping to digest, prioritize, and shape a better understanding of the broader spectrum of peer-reviewed evidence related to a specific clinical theme.

(C) Society of Surgical Oncology 2019

First Received: 4 December 2018; Published Online: 8 February 2019

R. S. Berman, MD e-mail: Russell.Berman@nyumc.org; russell.berman@nyulangone.org
The importance of systematic reviews in the oncologic literature was detailed in a recent commentary published in the Journal of the National Cancer Institute by Weed. ${ }^{6}$ In this commentary, Weed called for the submission of wellperformed systematic reviews in oncology, citing the ability of a systematic review (with or without meta-analysis) to increase transparency and clarity of research, and to help inform decisions and priorities. He also succinctly summarized the key components defining a systematic review as "a review with a clearly formulated research question that uses explicit methods to identify, select, and critically appraise relevant research and to collect and analyze data from the studies included in the review." In contrast to the less rigorous "narrative" review, the authors of a systematic review must rely upon method and not on their subjective judgment. Weed's succinct, well-written article illuminates the well-defined, peer-reviewed science dedicated to the writing and evaluation of review articles, and is a "must read" for those who use, author, and/or edit reviews.

In a 2008 Institute of Medicine report, it was determined that even when authors set out to perform a systematic review, they often fall short of their goal. ${ }^{7}$ To mitigate this potential pitfall, guidelines have been developed to facilitate the evaluation of systematic reviews. For example, PRISMA (Preferred Reporting Items for Systematic Reviews and Meta-Analyses) consists of a 27-item checklist aimed at improving the transparency of systematic reviews. ${ }^{8}$ Another tool, AMSTAR (A Measurement Tool to Assess Systematic Reviews), was designed to document the quality of systematic reviews. ${ }^{9}$ These tools can be used to assess whether "The Role of Completion Lymph Node Dissection for Sentinel Node-Positive 
Melanoma" by Hieken and colleagues contains the essential attributes of a transparent, high-quality systematic review article.

Our intent is not to detail all the factors that should be considered when the aforementioned tools are used. Nonetheless, brief mention of some of the key elements is warranted. These include a statement of the rationale and objectives, detailed methods, information sources, search and data collection process, and study characteristics, as well as a discussion that includes a summary of evidence, limitations, and conclusions. Importantly, the authors should analyze risk of bias at each step.

In their systematic review, Hieken and colleagues provide an excellent rationale and statement of objectives (i.e., to review the potential benefits and harms of CLND for SLN-positive melanoma patients). The methodology they used was similar to the rigorous methodologic approach used to inform the American College of Surgeons Operative Standards for Cancer Surgery, volume 2 (detailed in its Methodology Research Protocol chapter), ${ }^{10}$ and the clinical question addressed by Hieken and colleagues was based on Melanoma Key Question 2 in the same reference: Do patients with a positive SLN biopsy need a completion lymph node dissection? ${ }^{11}$ The authors were forthcoming regarding the limitations of the conclusions that may be drawn from their review.

Hieken and colleagues also satisfied most of the required AMSTAR elements. They presented an a priori research question and design. They performed a comprehensive literature search using at least two electronic sources and defined key words as well as Medical Subject Headings (MESH) terms. Following the AMSTAR guidelines, they also defined how publications were excluded (e.g., based on small sample size, inferior design, or lack of long-term follow-up evaluation).

The 47 articles formally included in the systematic review were assigned strength of recommendation based on the Grading of Recommendations Assessment, Development, and Evaluation (GRADE) system of evidence. Although the authors reported no conflicts of interest, they did not reflect on possible bias. Overall, this effort meets many of the criteria associated with a quality systematic review.

Once a systematic review is deemed high quality, it is reasonable to use such data as part of an overall thoughtful approach to inform clinical decision making. It is important to recognize, however, that a systematic review, no matter how well executed, may be of limited utility when the underlying data are lacking or the appropriate factors have been insufficiently studied.

Based on their systematic review, Heiken and colleagues concluded that the current evidence supports a "selective and individualized approach to the management of clinically node-negative SLN-positive melanoma patients." The authors also suggested that "for low-risk, low-volume micrometastatic disease, SLN biopsy may be both diagnostic and therapeutic, and close clinical followup with imaging or CLND are reasonable options for appropriately selected patients." It may be surmised that based on the available evidence, including completed trials, stronger or additional clinical recommendations cannot be supported. Nonetheless, it is likely that nearly all melanoma clinicians will opine, based on available data, that CLND can be omitted for "low-risk" patients.

These are promising times for the care of patients with regionally metastatic melanoma. Currently, as the evidence base continues to mature, we can use high-quality, transparent, systematic reviews such as the one by Heiken and colleagues to help guide our clinical decisions, recognizing that even well-conducted randomized, multicenter phase 3 clinical trials cannot address all current and future clinical issues.

Given the absence of any clear signal from MSLT-II that patients with high-risk SLN disease (e.g., "high" tumor burden or other potential descriptors) have a survival benefit after CLND, identifying those who may benefit is no small task. Clearly, several factors predict the risk of additional non-sentinel node involvement as well as the risk of death from melanoma and that our definition of "high" and "low" risk likely will continue to be refined. Further exploration of both clinicopathologic and potential molecular and immune-based factors in both tumor and the microenvironment may ultimately help to define risk further in such clinical scenarios. ${ }^{12}$ As the impact of clinical practice migration away from CLND continues to be broadly assessed in the setting of new and effective adjuvant-targeted $^{13}$ and checkpoint inhibitor-based therapies $^{14,15}$ for SLN-positive patients, these and other important clinical issues will no doubt continue to be explored and debated.

DISCLOSURE There are no conflicts of interest.

\section{REFERENCES}

1. Hieken TJ, Kane JM, Wong SL. The role of completion lymph node dissection for sentinel lymph node-positive melanoma. Ann Surg Oncol. 2019. https://doi.org/10.1245/s10434-018-6812-z.

2. Leiter U, Stadler R, Mauch C, et al. Complete lymph node dissection versus no dissection in patients with sentinel lymph node biopsy positive melanoma (DeCOG-SLT): a multicentre, randomised, phase 3 trial. Lancet Oncol. 2016;17:757-67.

3. Faries MB, Thompson JF, Cochran AJ, et al. Completion dissection or observation for sentinel-node metastasis in melanoma. N Engl J Med. 2017;376:2211-22.

4. Coit D. The enigma of regional lymph nodes in melanoma. $N$ Engl J Med. 2017;376:2280-1.

5. Rand JG, Faries MB. Omitting completion dissection in melanoma? Help is available for surgeons coping without routine 
dissection, but more work is needed. Ann Surg Oncol. 2018;25(12):3416-8. https://doi.org/10.1245/s10434-018-6744-7.

6. Weed DL. The need for systematic reviews in oncology. $J$ Natl Cancer Inst. 2018;110:812-4.

7. Institute of Medicine. Knowing what works in health care: a roadmap for the nation. The National Academies Press, Washington, DC, 2008.

8. Liberati A, Altman DG, Tetzlaff J, et al. The PRISMA statement for reporting systematic reviews and meta-analyses of studies that evaluate health care interventions: explanation and elaboration. PLoS Med. 2009;6(7):e1000100.

9. Shea BJ, Hamel C, Wells GA, et al. AMSTAR is a reliable and valid measurement tool to assess the methodological quality of systematic reviews. J Clin Epidemiol. 2009;62:1013-20.

10. Methodology research protocol. In: Katz M (ed) Operative standards for cancer surgery: esophagus, melanoma, rectum, stomach, thyroid, vol. 2. Wolters Kluwer, 2018.

11. Sentinel lymph node biopsy. In: Katz M (ed) Operative standards for cancer surgery: esophagus, melanoma, rectum, stomach, thyroid, vol. 2. Wolters Kluwer, 2018.
12. Gershenwald JE, Scolyer RA. Melanoma staging: American Joint Committee on Cancer (AJCC), 8th edition and beyond. Ann Surg Oncol. 2018;25:2105-10.

13. Long GV, Hauschild A, Santinami M, et al. Adjuvant dabrafenib plus trametinib in stage III BRAF-mutated melanoma. $N$ Engl $J$ Med. 2017;377:1813-23.

14. Weber J, Mandala M, Del Vecchio M, et al. Adjuvant nivolumab versus ipilimumab in resected stage III or IV melanoma. $N$ Engl $J$ Med. 2017;377:1824-35.

15. Eggermont AMM, Blank CU, Mandala M, et al. Adjuvant pembrolizumab versus placebo in resected stage III melanoma. $N$ Engl J Med. 2018;378:1789-801.

Publisher's Note Springer Nature remains neutral with regard to jurisdictional claims in published maps and institutional affiliations. 